\title{
Beyond The Skin: A Case Report of Vaginal Melanoma
}

\author{
Brittany Gilbert, DO', Tanner Gilbert, DO', Ardeshir Hakam, MD, MBA², \\ Lynne J. Goebel, MD, FACP1
}

ABSTRACT

Author affiliations are

listed at the end of this

Mucosal melanomas are rare, accounting for only $1.4 \%$ of all melanomas. Only article.

$18 \%$ of mucosal melanomas are vulvovaginal in origin, making it exceedingly rare. Mucosal melanomas typically carry a worse prognosis than those arising from cutaneous sites with a higher risk of recurrence and disseminated disease. We report a rare case of vaginal melanoma presenting as postmenopausal bleeding and discuss management of this disease.

Correspondence to: Lynne J. Goebel, MD Marshall Unviersity Joan C. Edwards School of Medicine goebel@marshall.edu

\section{KEYWORDS}

Vaginal Melanoma, Postmenopausal Vaginal Bleeding, Treatment

\section{INTRODUCTION}

In addition to arising from the skin, melanomas may arise from the mucosal lining of the respiratory, gastrointestinal, and genitourinary tracts, all of which contain melanocytes. Mucosal melanomas make up only about $1.4 \%$ of all melanomas in the United States. ${ }^{1}$ They are more common in women compared to men (2.8 per million vs. 1.5 per million) and in whites compared to blacks (2:1) when talking about absolute numbers of patients, but the racial difference is not as marked as with cutaneous melanoma (16:1). ${ }^{2}$ Mucosal melanomas account for $4.7 \%$ of melanomas occurring in black men compared to less than $1 \%$ of those occurring in white men. Likewise, mucosal melanomas make up $13.4 \%$ of melanomas in black women compared to $2.1 \%$ in white women. ${ }^{1}$ A study of ethnic Chinese seen at one institution in China noted $23 \%$ of their 522 cases of melanoma seen since 2006 were mucosal. ${ }^{3}$ Authors hypothesize that native Chinese may differ in risk to those who immigrated to the United States.

The most common sites of mucosal melanoma are the sinuses, oral cavity, vulvovaginal, and anorectal regions. ${ }^{2}$ Only $18 \%$ of mucosal melanomas are vulvovaginal in origin with $77 \%$ of these being vulvar and $20 \%$ being vaginal, making vaginal melanoma exceedingly rare. The etiology of mucosal melanomas is unclear, but it differs from cutaneous melanoma in that it is not UV light-induced. Evidence is lacking linking it to human papillomavirus, human herpes virus, or polyomavirus. ${ }^{2}$ Mucosal melanomas typically carry a worse prognosis than those arising from cutaneous sites with a higher risk of recurrence and disseminated disease. ${ }^{2}$

We report a rare case of vaginal melanoma causing postmenopausal bleeding and discuss the treatment of this disease.

\section{CASE}

An 80-year-old female presented to her gynecologist for vaginal bleeding. A pap smear was negative for cervical cancer. Saline infusion sonohysterography revealed a $6 \mathrm{~mm}$ endometrial stripe and an irregular polyploid lesion in the uterine cavity removed 
by dilation and curettage. The pathology of this lesion was benign. The patient reported no vaginal bleeding for two months but then it returned, much heavier than before. At this time exam revealed four lesions which were biopsied: two vulvar, one vaginal, and one periurethral. The pathology of the left vulvar lesion showed hyperpigmentation and a melanocytic macule. The pathology of the right vulvar lesion showed reactive changes. A shave biopsy of the polyploid lesion on the right side of the vaginal introitus revealed malignant melanoma. $A$ brush biopsy of the periurethral lesion also revealed malignant melanoma. A PET/CT scan showed hypermetabolic left groin nodes and increased uptake near the lesion at the vaginal introitus. The patient underwent wide local excision of the vagina and vulva and left inguinal node dissection. Two of the ten groin nodes were positive for melanoma. She was NRAS and c-KIT wild type (absent mutation). The periurethral lesion was a local metastasis. She received external beam and high dose vaginal brachytherapy and adjuvant chemotherapy with 5 cycles of cisplatin (6th cycle held due to increased creatinine) and 6 cycles of temozolomide completing treatment by March of 2014. There is no evidence of recurrence at five years. Figures 1 and 2 show the pathology.

\section{DISCUSSION}

We report a case of vaginal melanoma presenting as post-menopausal bleeding. Vaginal melanoma is rare with about 500 cases reported in the literature. ${ }^{4}$ The majority of vaginal melanoma cases occur in women with a mean age of 60 years. ${ }^{5}$ The most common presenting symptom is vaginal bleeding as in our patient. The lesion often appears as a mass, plaque, or ulceration and frequently involves the distal one-third of the anterior vaginal wall. ${ }^{6}$ Huang reported that ulcerative and nodular tumors have a better five-year survival rate than cauliflowerlike lesions with $40 \%, 38 \%$, and $20 \%$ survival respectively. Tumors less than three $\mathrm{cm}$ in size have a better prognosis with a median survival of 41 months compared to 12 months in tumors greater than three $\mathrm{cm} .{ }^{8}$ Unlike cutaneous melanoma, the thickness of the lesion is not linked to prognosis. Positive lymph nodes are associated with lower median overall survival (7.8 months compared to 30 months for node-negative disease). ${ }^{5}$ Similarly, the SEER (Surveillance, Epidemiology and End Results) database shows 1.98 times increased risk of death for vaginal melanoma patients with positive lymph nodes. ${ }^{9}$ Five-year survival rates for vaginal melanoma are between $5 \%-32.3 \% .{ }^{7,10,11}$ Our patient is still alive at five years which is unusual for a patient with regional metastasis.

Treatment plans should be individualized and are dependent upon the location, size, clinical stage, and tumor markers. Exactly which staging guide to use is unclear for vaginal melanoma as no staging method adequately predicts prognosis. Some clinicians use the FIGO (International Federation of Gynecology and Obstetrics) staging and others use

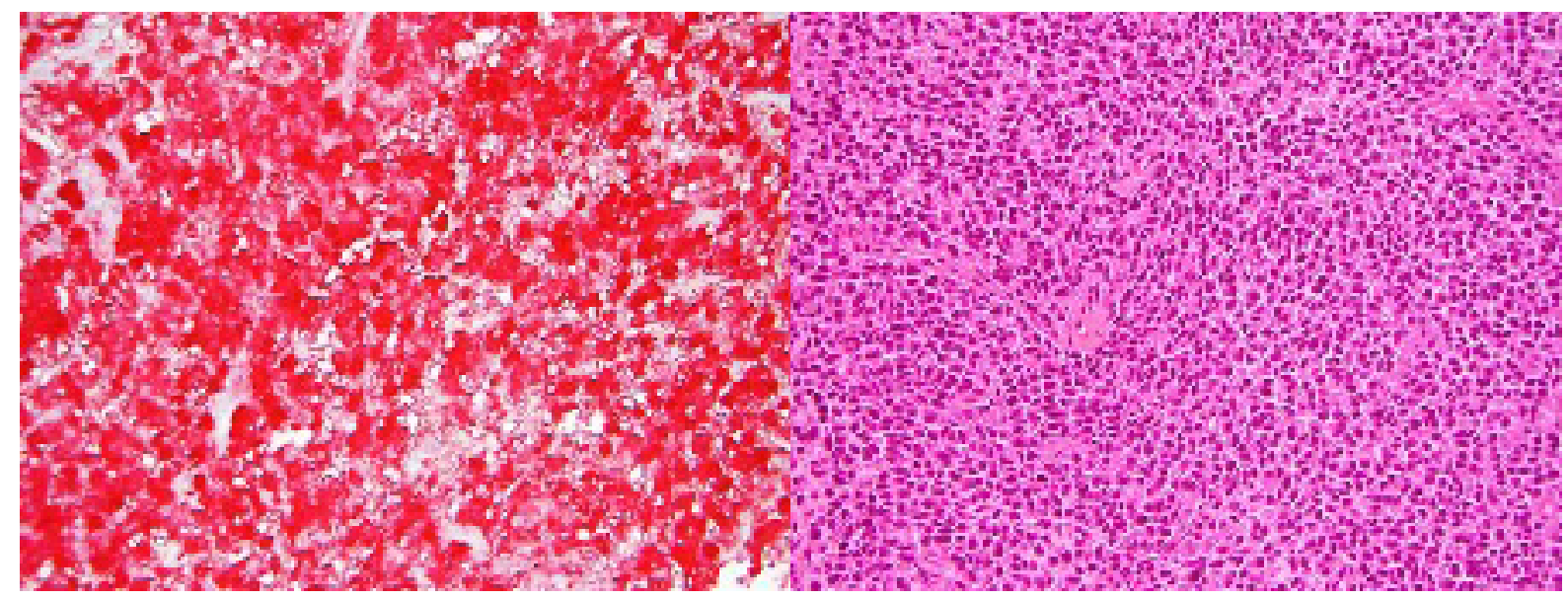

FIGURE 1. Mucosal melanoma involving vaginal epithelium stained with S100 for melanoma (left) and Hematoxylin and Eosin (right) (20x magnification). 


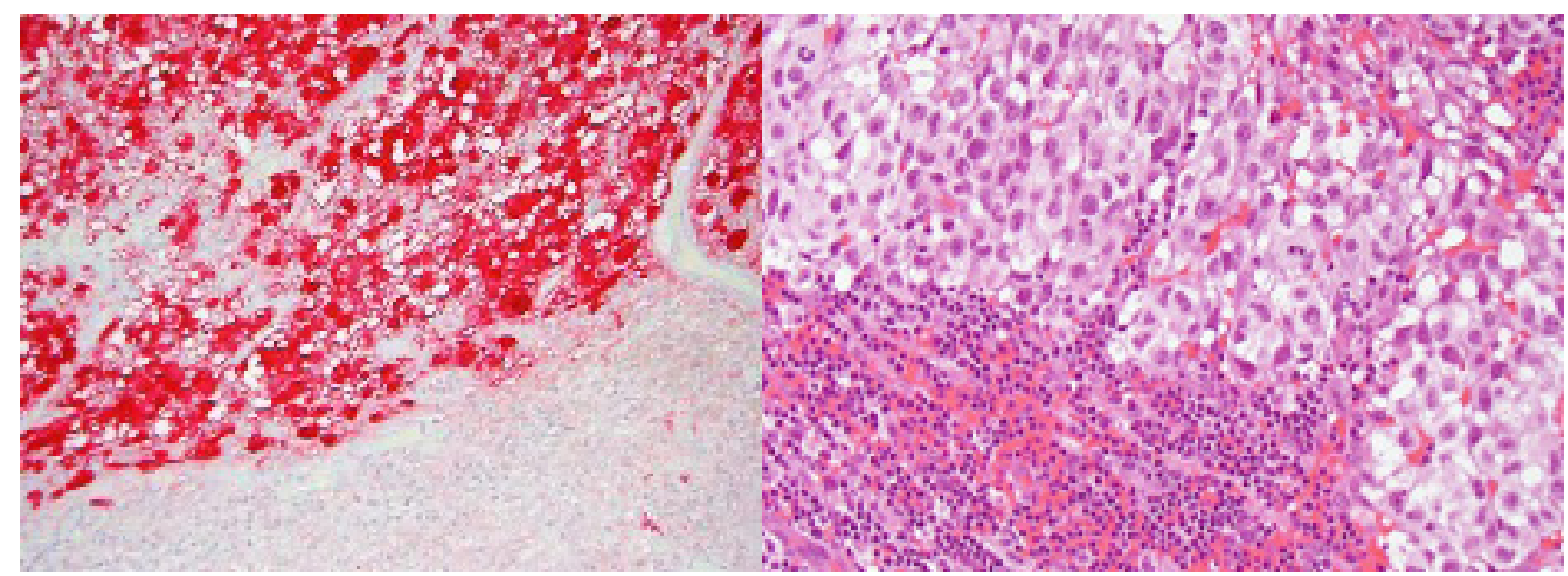

FIGURE 2. Lymph node stained with $\mathrm{S} 100$ for melanoma (left) and Hematoxylin and Eosin (right) (20x magnification).

the AJCC (American Joint Commission on Cancer). In their review article, Gaducci et al stated that the AJCC system is recommended for vulvar and vaginal melanomas and the FIGO system for cervical melanomas. ${ }^{4}$

Wide local excision offers patients the best chance of long-term survival. ${ }^{5}$ Adjuvant radiation can be used as in our patient for local disease control, but the evidence is lacking for its efficacy at increasing survival. ${ }^{9}$ A study out of MD Anderson reported less local recurrence and increased median overall survival with adjuvant radiotherapy after wide local excision (from 16.1 months to 29.4 months) but it was not statistically significant. ${ }^{5}$ Carbon ion beams could be useful in vulvovaginal melanomas with lymph node metastasis in the pelvis and groin with three-year local control rates of $49.9 \%$ and overall survival of $53.0 \% .^{12}$

Limited data exist on the use of adjuvant systemic therapy in patients with mucosal melanoma because of difficulty enrolling patients in clinical trials. In one study, postoperative chemotherapy with temozolomide plus cisplatin, similar to the regimen our patient received, significantly prolonged median relapse-free survival when compared to interferonalpha- $2 \mathrm{~b}$ alone or surgery alone, but there were only 21 patients with vulvar and 19 with vaginal disease included in this study. ${ }^{13}$ Temozolomide plus cisplatin had the best outcome for overall survival of 48.7 months versus 40.4 months with high dose interferon. Immunotherapy with IL-2, interferon- alpha, BCG, dendritic cells or lymphokine-activated killer cells may be beneficial as adjuvant therapy to surgery however this recommendation was from a retrospective study of mainly early-stage disease. ${ }^{7}$ BRAF (encodes for protein B-Raf serine/threonine kinase), KIT (encodes for receptor tyrosine kinase protein) and NRAS (encodes a protein N-Ras that acts as a switch for receptor tyrosine kinase) are oncogenes with the potential to cause normal cells to become cancerous and treatment may target them. BRAF is a common mutation found in about half of cutaneous melanomas, ${ }^{14}$ however only 10 percent of mucosal melanomas have mutations of BRAF, and approximately 25 percent have amplifications or somatic mutations of KIT. ${ }^{15,16,17}$ NRAS mutations may be found more often in vaginal as opposed to vulvar melanomas suggesting that these two anatomic locations may be immunologically different. ${ }^{4}$ Hou et al studied 51 cases of vulvar and vaginal melanoma samples obtained from multiple institutions and found $22 \%$ were positive for c-KIT compared to 3.5\% of cutaneous and other nongynecological mucosal melanomas. ${ }^{18}$ They also found a difference in vulvar and vaginal melanomas with more KIT mutations in the vulvar (26.5\%) compared to vaginal (8.3\%) location. Hou et al found BRAF mutations in $26 \%$ of vulvovaginal melanomas. This is higher than seen in other series such as Aulmann et al who reported no BRAF mutations in 39 vulvar and 15 vaginal melanomas. ${ }^{19}$ Hou et al explains this difference by stating that her samples were oftentimes from metastatic lesions which may differ in frequency of mutation from primary 
lesions. ${ }^{18}$ Half of the BRAF mutations in Hou's group were of the $\mathrm{V} 600 \mathrm{E}$ type indicating a response to the drug vemurafenib, a selective inhibitor of the $\mathrm{V} 600 \mathrm{E}$ mutant BRAF kinase. NRAS mutations were rare in this group of vulvovaginal melanomas (4\%). PD-1 (programmed cell death) and PD-L1 (programmed cell death ligand) were seen in $75 \%$ and $56 \%$ of vulvovaginal melanomas in this series.

In patients with metastatic cutaneous melanoma and a BRAF V600E/K mutation, the combination of a BRAF inhibitor (ex. vemurafenib, dabrafenib) and a MEK inhibitor (ex. cobimetinib, trametinib) has a longer progression-free survival, higher objective response rate, and longer overall survival compared with a BRAF inhibitor alone. ${ }^{20-22}$ For metastatic cutaneous, mucosal, and acral melanoma patients with a KIT mutation, the use of imatinib, a c-KIT inhibitor, in a phase II trial demonstrated better disease control however phase III data is not available. ${ }^{23}$ Two other studies suggest imatinib may be helpful in metastatic mucosal melanoma with KIT mutations, but the number of mucosal melanoma patients in the studies was small limiting the strength of the recommendation. ${ }^{15,24}$ In eleven vulvovaginal melanoma patients who were not surgical candidates, different combinations of biochemotherapy achieved a partial response with a median survival of ten months. ${ }^{25}$ The combination of anti-PD-1 (ex. pembrolizumab, nivolumab) and antiCTLA4 (cytotoxic T lymphocyte-associated protein) (ex. ipilimumab) agents was more beneficial than single-agent anti-PD-1 therapy in a pooled analysis of metastatic mucosal melanoma patients, however, median progression-free survival only improved by 2.9 months with $40 \%$ having grade 3 or 4 toxicity. ${ }^{26}$

There are no consensus guidelines for the treatment of mucosal melanoma but an expert panel believes that it should be treated the same as cutaneous melanoma due to a lack of clinical trial data specific to mucosal disease. ${ }^{27}$ Cutaneous melanoma treatment has changed significantly since the introduction of immune checkpoint inhibitors and targeted therapy based on genetic mutations with exciting improvements in outcomes. Future treatments for mucosal melanomas may diverge from cutaneous disease because we have evidence that the disease sites differ in the frequency of genetic mutations with implications for targeted therapy.

\section{CONCLUSION}

We report a case of vaginal melanoma presenting with postmenopausal bleeding to raise awareness for this rare disease. Despite aggressive therapy, prognosis remains poor for most patients with vaginal melanoma. The key to improved survival is early detection and wide local excision of the lesion. We report this rare disease so physicians will consider it in their differential diagnosis for vaginal bleeding and perform repeat examinations when the usual evaluation and treatment for bleeding is not successful. Advances in immunotherapy and targeted therapy for vaginal melanoma may offer specific treatment options, however, until more evidence is available, the current recommendation is to treat with similar regimens used in cutaneous melanoma or offer enrollment in clinical trials.

\section{AUTHOR AFFILIATIONS}

1. Marshall University Joan C. Edwards School of Medicine, Huntington, West Virginia

2. Moffitt Cancer Center, Tampa, Florida

\section{REFERENCES}

1. McLaughlin CC, Wu XC, Jemal A, Martin HJ, Roche LM, Chen VW. Incidence of noncutaneous melanomas in the U.S. Cancer. 2005;103:1000-7.

2. Mihajlovic M, Vlajkovic S, Jovanovic P, Stefanovic V. Primary mucosal melanomas: a comprehensive review. Int J Clin Exp Pathol. 2012;5(8):739-753.

3. Chi Z, Li S, Sheng X, et al. Clinical presentation, histology, and prognoses of malignant melanoma in ethnic Chinese: a study of 522 consecutive cases. BMC Cancer. 2011;11:85.

4. Gadducci A, Carinelli S, Guerrieri ME, Aletti GD. Melanoma of the lower genital tract: Prognostic factors and treatment modalities. Gynecol Oncol. 2018;150(1):180-189.

5. Frumovitz M, Etchepareborda M, Sun CC, et al. Primary malignant melanoma of the vagina. Obstet Gynecol. 2010;116:1358.

6. DeMatos P, Tyler D, Seigler HF. Mucosal 
melanoma of the female genitalia: a clinicopathologic study of forty-three cases at Duke University Medical Center. Surgery. 1998; 124:38.

7. Huang Q, Huang H, Wan T, Deng T, Liu J. Clinical outcome of 31 patients with primary malignant melanoma of the vagina. J Gynecol Oncol. 2013;24:330-335.

8. Buchanan DJ, Schlaerth J, Kurosaki T. Primary vaginal melanoma: thirteen-year disease-free survival after wide local excision and review of recent literature. Am J Obstet Gynecol. 1998;178(6):1177-1184.

9. Kirschner AN, Kidd EA, Dewees T, Perkins SM. Treatment approach and outcomes of vaginal melanoma. Int J Gynecol Cancer. 2013;23:14841489.

10. Verschraegen CF, Benjapibal M, Supakarapongkul W, et al. Vulvar melanoma at the M. D. Anderson Cancer Center: 25 years later. Int J Gynecol Cancer. 2001;11:359.

11. Creasman WT, Phillips JL, Menck HR. The National Cancer Data Base report on cancer of the vagina. Cancer. 1998;83:1033.

12. Karasawa K, Wakatsuki M, Kato S, Kiyohara H, Kamada T. Working group for gynecological tumors. Clinical trial of carbon ion radiotherapy for gynecological melanoma. J Radiat Res. 2014;55:343-350.

13. Lian B, Si L, Cui C, et al. Phase II randomized trial comparing high-dose IFN-a2b with temozolomide plus cisplatin as systemic adjuvant therapy for resected mucosal melanoma. Clin Cancer Res. 2013;19:4488.

14. Bhatia P, Friedlander P, Zakaria E, Kandil E. Impact of BRAF mutation status in the prognosis of cutaneous melanoma: an area of ongoing research. Ann Transl Med. 2015;3(2):24.

15. Carvajal RD, Antonescu CR, Wolchok JD, et al. KIT as a therapeutic target in metastatic melanoma. JAMA. 2011;305:2327.

16. Curtin JA, Busam K, Pinkel D, Bastian BC. Somatic activation of KIT in distinct subtypes of melanoma. J Clin Oncol 2006;24:4340.

17. Beadling C, Jacobson-Dunlop E, Hodi FS, et al. KIT gene mutations and copy number in melanoma subtypes. Clin Cancer Res. 2008;14:6821-6828.

18. Hou JY, Baptiste C, Hombalegowda RB, et al. Vulvar and vaginal melanoma: a unique subclass of mucosal melanoma based on a comprehensive molecular analysis of 51 cases compared with 2253 cases of nongynecologic melanoma. Cancer. 2017;123:1333-44.

19. Aulmann S, Sinn HP, Penzel R, et al. Comparison of molecular abnormalities in vulvar and vaginal melanomas Mod Pathol. 2014;27:1386-1393.

20. Long GV, Stroyakovskiy D, Gogas $\mathrm{H}$, et al. Combined BRAF and MEK inhibition versus BRAF inhibition alone in melanoma. $\mathrm{N}$ Engl J Med. 2014;371:1877.

21. Long GV, Stroyakovskiy D, Gogas $\mathrm{H}$, et al. Dabrafenib and trametinib versus dabrafenib and placebo for Val600 BRAF-mutant melanoma: A multicentre, double-blind, phase 3 randomised controlled trial. Lancet. 2015; 386:444.

22. Long GV, Flaherty KT, Stroyakovskiy D, et al. Dabrafenib plus trametinib versus dabrafenib monotherapy in patients with metastatic BRAF V600E/K-mutant melanoma: Long-term survival and safety analysis of a phase 3 study. Ann Oncol. 2017;28:1631.

23. Hodi FS, Corless CL, Giobbie-Hurder A, et al. Imatinib for melanomas harboring mutationally activated or amplified KIT arising on mucosal, acral, and chronically sun-damaged skin. J Clin Oncol. 2013;31:3182-3190.

24. Guo J, Si L, Kong Y, et al. Phase II open-label, single-arm trial of imatinib mesylate in patients with metastatic melanoma harboring c-KIT mutation or amplification. J Clin Oncol. 2011;29:2904-2909.

25. Harting MS, Kim KB. Biochemotherapy in patients with advanced vulvovaginal mucosal melanoma. Melanoma Res. 2004;14:517-520.

26. D'Angelo SP, Larkin J, Sosman JA, et al. Efficacy and safety of nivolumab alone or in combination with Ipilimumab in patients with mucosal melanoma: a pooled analysis. J Clin Oncol. 2017;35:226.

27. Seth R, Messersmith $H$, Kaur V et al. Systemic therapy for melanoma: ASCO Guideline. J Clin Oncol. 2020; 31:JCO2000198. 